\title{
Investigation of the human pathogen Acinetobacter baumannii under iron limiting conditions
}

\author{
Bart A Eijkelkamp', Karl A Hassan², lan T Paulsen², Melissa H Brown ${ }^{1 *}$
}

\begin{abstract}
Background: Iron acquisition systems are important virulence factors in pathogenic bacteria. To identify these systems in Acinetobacter baumannii, the transcriptomic response of the completely sequenced strain ATCC 17978 under iron limiting conditions was investigated using a genomic microarray that contained probes for all annotated open reading frames.

Results: Under low iron conditions, transcription levels were more than 2-fold up-regulated for 463 genes, including 95 genes that were up-regulated more than 4-fold. Of particular significance, three siderophore biosynthesis gene clusters, including one novel cluster, were highly up-regulated. Binding sites for the ferric uptake regulator were identified in the promoter regions of many up-regulated genes, suggesting a prominent role for this regulator in the Acinetobacter iron acquisition response. Down-regulation under iron limitation was less dramatic as the transcription of only 202 genes varied more than 2-fold. Various genes involved in motility featured prominently amongst the genes down-regulated when iron was less readily available. Motility assays confirmed that these transcriptional changes are manifested at the phenotypic level. The siderophore biosynthesis gene clusters were further investigated by means of comparative genomic analysis of 10 sequenced Acinetobacter isolates. These analyses revealed important roles for mobile genetic elements in shaping the siderophore meditated iron acquisition mechanisms between different Acinetobacter strains.

Conclusions: A. baumannii grown under iron limited conditions resulted in major transcriptional changes of not only many iron acquisition related genes, but also genes involved in other processes such as motility. Overall, this study showed that A. baumannii is well adaptable to growth in an environment which has limiting iron availability.
\end{abstract}

\section{Background}

An increasing prevalence of infections caused by Acinetobacter baumannii has been observed in the clinical setting throughout the last 10 to 15 years [1,2]. A. baumannii is able to persist in the hospital environment and in particular intensive care units, due to its wide variety of resistance mechanisms and high survival rate on abiotic surfaces [3-6]. Some clinical A. baumannii strains have been shown to be naturally competent for the uptake of genetic material, which facilitates acquisition of novel resistance and virulence genes [7-9].

\footnotetext{
* Correspondence: melissa.brown@flinders.edu.au

'School of Biological Sciences, Flinders University, Adelaide, SA 5001, Australia

Full list of author information is available at the end of the article
}

Free iron is a limited micronutrient in hosts where it is typically tightly bound within a range of biomolecules, such as heme. As such, iron acquisition systems are important factors for the virulence of pathogenic organisms. Bacteria can adapt to iron limited host environments through the expression of a range of iron acquisition mechanisms. One pathway for uptake of iron involves direct binding of $\mathrm{Fe}^{2+}$ or heme to receptors or transport proteins on the cell surface [10]. A second more energy intensive mechanism of iron uptake involves the production and secretion of high-affinity iron chelating siderophores, which compete with host cells for iron $[11,12]$. The genes involved in the production of a siderophore are usually clustered within the genome of the producing organism. In addition to biosysnthesis genes, many of these gene clusters also
C Biomed Central

() 2011 Eijkelkamp et al; licensee BioMed Central Ltd. This is an Open Access article distributed under the terms of the Creative Commons Attribution License (http://creativecommons.org/licenses/by/2.0), which permits unrestricted use, distribution, and reproduction in any medium, provided the original work is properly cited. 
encode efflux pumps with putative roles in siderophore export. Transporters classified within the ATP-binding cassette (ABC) superfamily, major facilitator superfamily (MFS) and resistance-nodulation-cell division (RND) family have been associated with siderophore extrusion [13]. However, the ability to transport siderophores into the extracellular space has been shown for only two pumps, both MFS members. EntS of Escherichia coli transports enterobactin [14] and YmfE of Bacillus subtilis is involved in transport of bacillibactin [15]. Inactivation of these pumps results in decreased efflux of the fully synthesized siderophore, but increased extrusion of siderophore precursor products $[14,15]$.

The uptake and reduction of iron-loaded ferric siderophores involves the TonB-ExbB-ExbD energy transduction system in combination with a ferric siderophore complex receptor [16,17]. Bacteria often contain numerous ferric siderophore complex receptors. Some of these are encoded within siderophore biosynthesis gene clusters and are likely to display specificity for the locally encoded siderophore. However, various other ferric siderophore receptors can be scattered throughout the genome and may have the ability to recognize exogneously produced siderophores that are structurally unrelated to endogenous siderophores $[18,19]$.

Most A. baumannii strains have the ability to grow under iron limiting conditions, which assists in the colonization of a host, however, a diversity of iron acquisition mechanisms has been shown between different Acinetobacter strains [20]. To date, three different siderophore biosynthesis gene clusters have been described in $A$. baumannii [21-23]. Of these, the cluster encoding the siderophore acinetobactin has been the most extensively studied. Knockout experiments have confirmed the functions of both a siderophore biosynthesis protein and receptor from the acinetobactin gene cluster [24]. Furthermore, acinetobactin is the only siderophore produced by Acinetobacter to have been structurally characterized [25]. A second siderophore biosynthesis gene cluster found only in A. baumannii 8399 has been characterized using complementation experiments in an $E$. coli mutant strain [21]. Finally, a putative siderophore biosynthesis gene cluster has been described in strain ATCC 17978 and subjected to limited quantitative reverse transcription PCR (qRT-PCR) analyses under iron limiting conditions [23].

To comprehensively identify mechanisms of iron acquisition and low iron adaptation in A. baumannii, the affect of iron starvation on A. baumannii cells was investigated at the global level. The transcriptomic responses of A. baumannii ATCC 17978 cells to low iron conditions were examined using a whole genome microarray.

\section{Results and Discussion}

\section{Optimization of test conditions for transcriptomics}

Iron is an essential micronutrient and depletion in the growth medium is likely to have an impact on cell viability. Therefore, growth of A. baumannii ATCC 17978 was investigated under varying iron concentrations to determine optimal conditions for whole transcriptome analysis. Reduction of available iron in Mueller-Hinton (MH) medium was achieved by supplementation of 2,2'dipyridyl (DIP), a synthetic iron chelator. This compound had no effect on the $\mathrm{pH}$ of the medium (data not shown). No significant change in the growth rate of $A$. baumannii strain ATCC 17978 was observed after addition of 100 $\mu \mathrm{M}$ DIP, whereas, supplementation with $200 \mu \mathrm{M}$ DIP resulted in a growth delay of approximately $45 \mathrm{~min}$ at mid-log phase $\left(\mathrm{OD}_{600}=0.7\right)$ (Figure 1$)$. Moreover, the total biomass was reduced by more than $10 \%$ at stationary phase (>240 min). Addition of $300 \mu \mathrm{M}$ DIP had a major impact on growth and resulted in more than $70 \%$ biomass reduction compared to cultures under iron replete conditions during stationary phase. Due to the moderate inhibitory, but non-lethal effect of $200 \mu \mathrm{M}$ DIP, this concentration was chosen to study transcriptional changes under iron limitation. Preliminary qRTPCR was performed to confirm transcriptional adaptation in response to iron limitation by assaying the level of transcription of the ferric uptake regulator (FUR), which is known for auto-up-regulation when iron is limited [26]. A. baumannii grown in the presence of $200 \mu \mathrm{M}$ DIP expressed FUR at levels increased more than 2-fold compared to cells grown under iron replete conditions (data not shown). Therefore, the genome wide transcriptional changes of ATCC 17978 grown in MH medium and MH

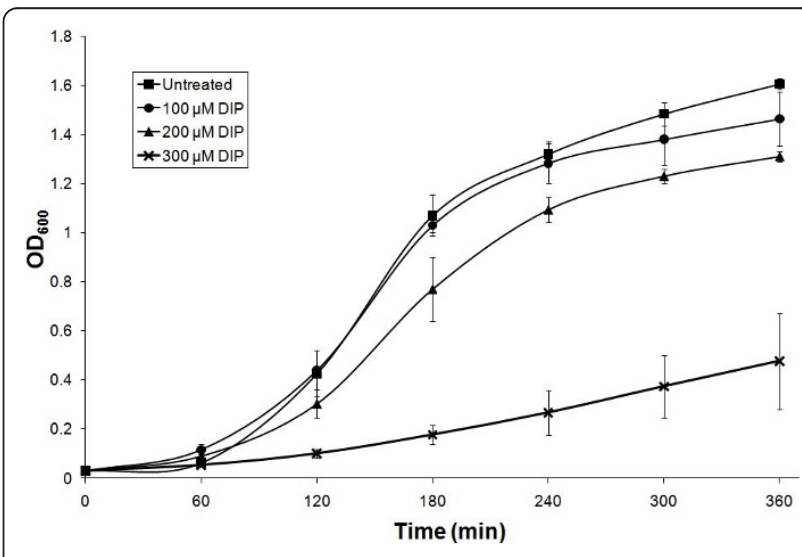

Figure 1 Growth curves of $A$. baumannii with varying iron concentrations. Growth under different iron concentrations was tested in Mueller-Hinton (MH) broth and $\mathrm{MH}$ supplemented with 2,2'-dipyridyl (DIP) to final concentrations of 100, 200 and $300 \mu \mathrm{M}$. Absorbance was measured every hour at $\mathrm{OD}_{600}$ for 6 hours; the data represent the average of three separate experiments and the error bars show the standard deviation. 
supplemented with $200 \mu \mathrm{M}$ DIP during mid log-phase were compared by microarray analysis.

\section{Global transcriptional changes of $A$. baumannii to iron starvation}

Iron limitation had far reaching transcriptional effects on A. baumannii cells (Figure 2). Significance analysis [27] of the microarray data showed that 1207 genes were significantly differentially expressed under iron limiting as compared to iron replete conditions (Additional file 1). Transcript levels were more than 2 -fold higher for 463 genes, of which 95 genes were upregulated more than 4 -fold (Figure 2 ). The maximum overexpression observed was 165 -fold for the siderophore biosynthesis gene basD. Fewer genes were downregulated under iron limitation; only 202 genes were more than 2-fold underexpressed with a maximum down-regulation of 29-fold (A1S_2297). The array results were validated by qRT-PCR analysis on a subset of differentially expressed genes (Figure 3 ). There was

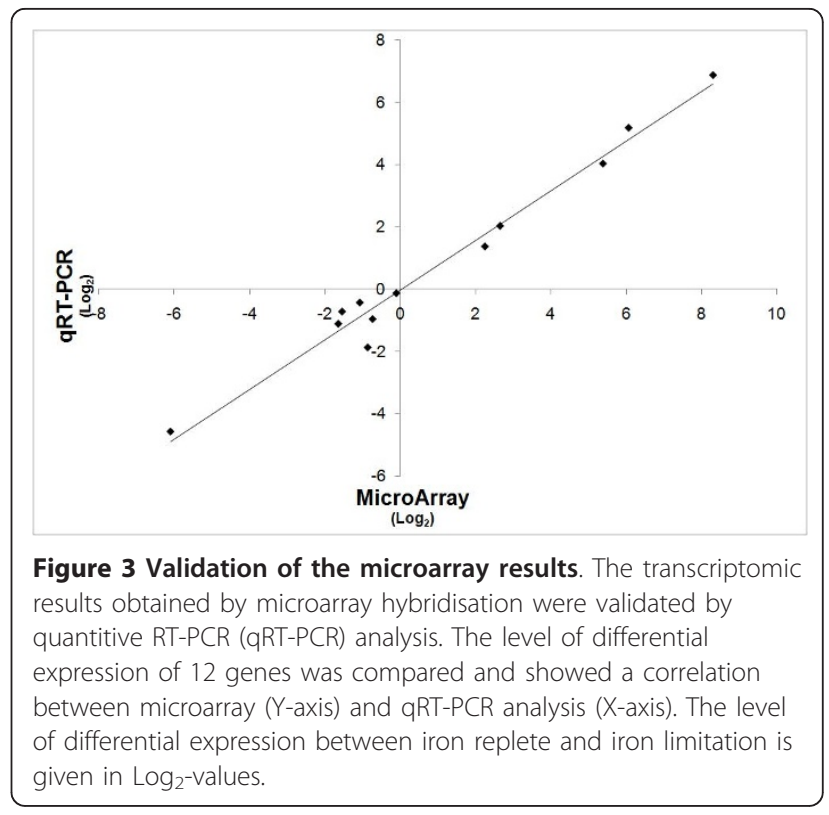

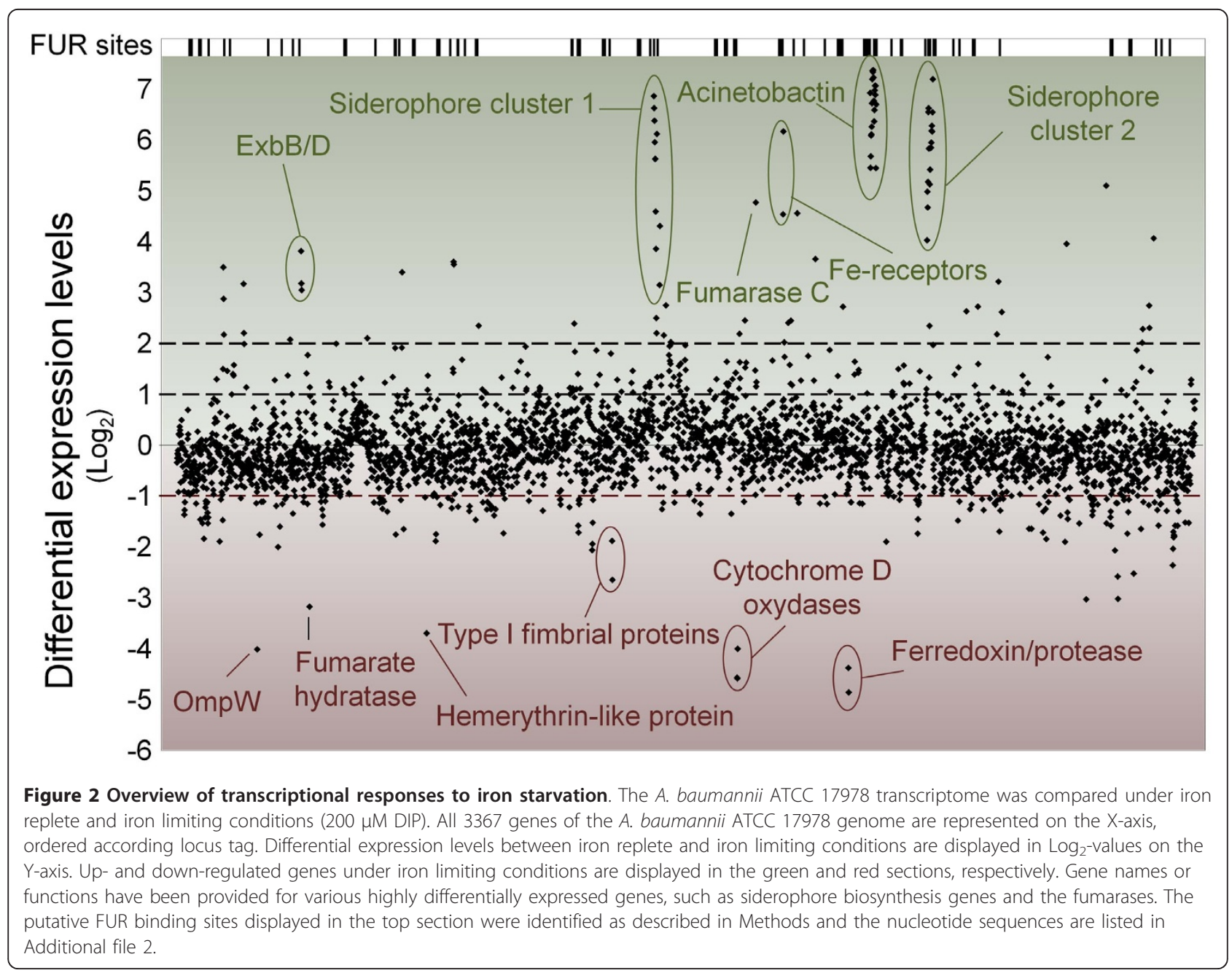


good correlation between data from the qRT-PCR and the microarray analyses, although the qRT-PCR data generally showed higher fold changes than the microarray expression data. The tendency for microarrays to underestimate fold changes relative to qRT-PCR is well established [28].

Microarray data displayed by clusters of orthologous groups (COG) functional categories showed that $27 \%$ of the genes up-regulated under iron limited conditions encode proteins involved in secondary metabolite biosynthesis, transport and catabolism (Figure 4). The majority of these genes are located within three large overexpressed gene clusters, each of which is known or predicted to synthesize a siderophore (Figure 2). Siderophore cluster 1 (A1S_1647-1657) is a novel putative siderophore gene cluster, having not been previously identified. The two other highly overexpressed siderophore clusters identified, siderophore cluster 2 (A1S_2562-2581) and the acinetobactin cluster (A1S_2372-2392), have been described previously
$[21,23]$. Many other overexpressed genes within this COG category encode ferric siderophore receptors, which are widely dispersed across the genome.

In addition to siderophore-related genes, a high percentage, $22 \%$, of genes up-regulated under iron limitation, encoded proteins categorized within the defence mechanism COG. A number of these genes encode transporter proteins that are classified as defence proteins due to their predicted roles in the export of metabolic waste or other toxic compounds. Interestingly, our analysis of several of these proteins, primarily those encoded within siderophore biosynthetic loci, suggested that they function in the extrusion of siderophores (Hassan et al., unpublished data).

Various genes related to cell motility were downregulated when $A$. baumannii was grown under iron limiting conditions (Figure 4). These included biosynthesis genes homologous to both type IV pili and chaperone-usher pili assembly systems, or type I pili. Another heavily down-regulated gene encoded a hemerythrin-like

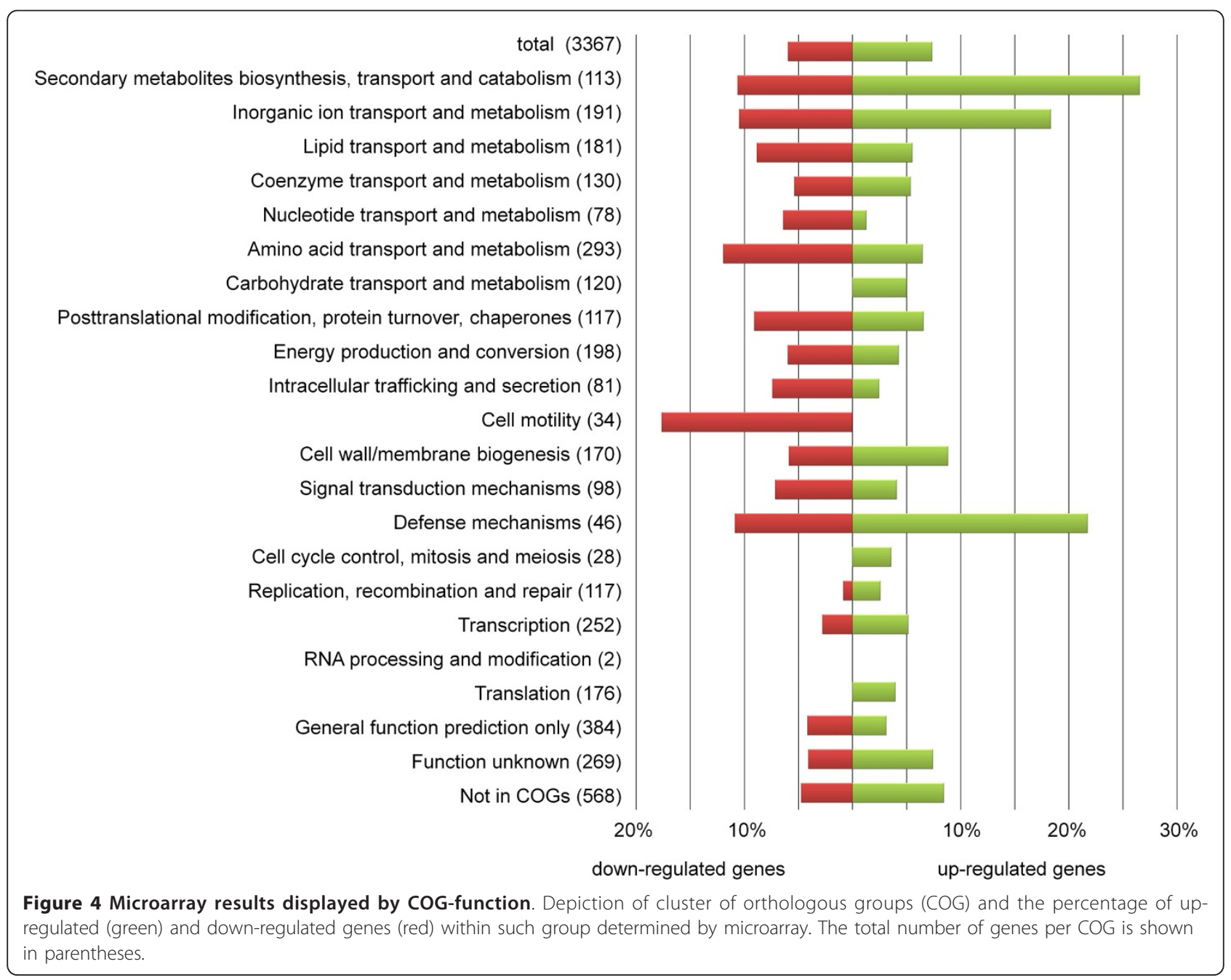


protein (A1S_0891). Various functions have been suggested for these proteins with an iron containing centre, including detoxification, transport and storage of iron and/or oxygen, or a role as a sensory protein [29]. The cytochrome D genes, part of the respiratory system, and $4 \mathrm{Fe}-4 \mathrm{~S}$-ferredoxin, which facilitates electron transport in various metabolic processes, were also down-regulated more than 4-fold. The iron-dependent Class I fumarate hydratase was found to be 9-fold down-regulated. In contrast, fumarase $\mathrm{C}$, which belongs to the iron-independent Class II was 27-fold up-regulated, suggesting a physiological shift to the Class II protein under iron limitation (Figure 2). These findings correlated with results observed in a study on fumarase A (Class I) and fumarase $\mathrm{C}$ in the tri-carboxylic acid cycle of Pseudomonas fluorescence [30]. In conclusion, A. baumannii showed strong transcriptional responses to iron starvation, predominantly in up-regulation of iron acquisition mechanisms. However, many genes related to other processes than iron acquisition, such as respiration and motility, were also transcriptionally affected under the conditions tested.

\section{A. baumannii FUR box optimization}

Bioinformatic analyses were performed to identify motifs within the promoter regions of iron responsive genes that could serve regulatory functions. The multiple em for motif elicitation (MEME) tool and the multiple alignment and search tool (MAST) were used to identify motifs and search for these motifs across the A. baumannii ATCC 17978 genome, respectively [31,32]. MEME-based analyses of the upstream regions of all genes overexpressed by at least 4-fold under iron limitation identified a motif bearing strong similarity to the FUR binding sites of E. coli and Pseudomonas spp. [33]. To confirm the importance of FUR in regulating iron adaptation responses in A. baumannii, a scoring matrix was created using the experimentally determined $E$. coli FUR binding sites [34]. This scoring matrix was used to screen the ATCC 17978 genome using MAST. Hits obtained using MAST, which were found upstream of genes that were more than 4-fold up-regulated in response to iron limitation and with a p-value less than $10 \mathrm{e}^{-5}$, were selected for iterative refinement of the scoring matrix, until no new hits were obtained (Table 1). The resulting A. baumannii FUR box motif showed a 25 nucleotide palindromic sequence (Figure 5).

In a previous study, the upstream regions of FUR genes from different $A$. baumannii isolates were aligned to obtain a motif representing the FUR binding site [26]. Some differences exist between this sequence and the FUR box sequence shown in Figure 5, most notably, the previously described motif lacked the typical FUR box palindomic structure. FUR is known to auto-regulate its
Table 1 Putative FUR binding sequences in the ATCC 17978 genome

\begin{tabular}{|c|c|}
\hline Locus tag & Putative FUR binding sequence $\left(5^{\prime}-3^{\prime}\right)$ \\
\hline A1S_0242 & TTATTTGGTAATTATTCTCATTTAT \\
\hline A1S_0416 & GGATTTGTTAATGATTATCATTTGC \\
\hline A1S_0474 & GCGAATAATAATAATTCTTATTTAT \\
\hline A1S_0980 & GATATTGTTAATAATTATCATTATT \\
\hline A1S_1647 & TGAAATGATAATAATTATCATTAAT \\
\hline A1S_1657 & ATAATTGATAATGATAATCATTITT \\
\hline A1S_1667 & GATAATGTAAATAATTCTCATTTAT \\
\hline A1S_2077 & TCATTTGATACTGATTATCAATATT \\
\hline A1S_2080 & ATAAATGAGAATGATTTTAATTAAT \\
\hline A1S_2123 & GATAATAAGAATTATTITTATTTGT \\
\hline A1S_2278 & TTATTTGATAATGATTTTCATTTAT \\
\hline A1S_2372 & GTTATTGATAATAATAATCATTTGC \\
\hline A1S_2382 & GCAACTGGTAATCATTTTCATTTGT \\
\hline A1S_2391 & GTAATTGTAAATGATTATCATTTAT \\
\hline A1S_2392 & GTAAATAATAATCATTATTAATTGT \\
\hline A1S_2567 & TTACTTGAGAATGATTCTTGATAAC \\
\hline A1S_2581 & TTAAATGAGAATCATTTTCATTTAT \\
\hline A1S_2582 & TTAAATGAGAATCATTTCATTTAT \\
\hline A1S_2667 & TTITTGAGAATTATTATTGATTAT \\
\hline A1S_3174 & ATTATTGATAATTATTATCGTTTGT \\
\hline A1S_3324 & GATAATGAGAATTATTTAAATTAT \\
\hline A1S_3339 & TTAAATGATTATAATTATCATTTAT \\
\hline
\end{tabular}

own expression, however, as seen in the current study, up-regulation of FUR under iron limiting conditions is at lower levels than that of other FUR regulated genes, e.g., siderophore biosynthesis genes, a phenomenon that could reflect a lower binding affinity of the FUR protein for the FUR promoter region [35-38]. Therefore, the FUR binding sequences found upstream of the FUR gene may have predicted a less than optimal A. baumannii FUR box consensus motif, which does not show the typical palindromic structure. Moreover, FUR motifs of different bacterial genera show a high level of homology, whereas the previously described $A$. baumannii FUR motif is more distant.

A MAST search (parameters; $\mathrm{E}<100, \mathrm{p}<10 \mathrm{e}^{-4}$ ) using the optimized $A$. baumannii FUR motif showed 81 hits

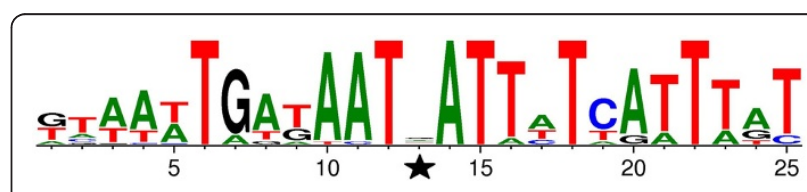

Figure 5 The optimized $A$. baumannii FUR motif. Per position, the size of the nucleotide ( $T$ in red, $A$ in green, $C$ in blue and $G$ in black) indicates its prevalence in the 22 included sequences from Table 1. The motif shows a palindrome with a central nonconserved nucleotide in position 13 which is indicated by the star. The figure of the A. baumannii ATCC 17978 FUR motif was created using WebLogo 3.0 [64]. 
to the A. baumannii ATCC 17978 genome (Additional file 2). Over $80 \%$ of the genes with a well conserved FUR box upstream $\left(\mathrm{p}<10 \mathrm{e}^{-5}, \mathrm{n}=41\right)$ showed more than 2-fold up-regulation. Furthermore, of the 95 genes up-regulated more than 4-fold under iron limiting conditions, 75 were preceded by putative FUR binding sites. These studies highlight a significant correlation between the level of conservation of a putative FUR binding site and the level of up-regulation under iron limiting conditions.

Extracytoplasmic function (ECF) transcription factors are sigma-70 family proteins that are responsive to environmental changes such as iron starvation [39]. These proteins play an important role in regulating iron-uptake mechanisms in several bacterial genera. For example, one of the best characterized ECF sigma factors, PvdS, controls the genes required for biosynthesis and transport of the siderophore pyoverdine in P. aeruginosa [40]. Expression of $p v d S$ and various other sigma70 factors in Pseudomonas is regulated by FUR [40]. However, in this study no predicted sigma-70 factors were identified in the list of genes containing a putative A. baumannii FUR binding site (Additional file 2). Moreover, no significant differentially expressed sigma70 factors were found under the iron limited conditions, suggesting that in Acinetobacter, these proteins do not function in iron-uptake regulation. Another regulatory mechanism involved in iron homeostasis includes small RNA molecules, such as $r y h B$ from $E$. coli or prrF from $P$. aeruginosa. However, sequences homologous to either of these small RNAs were not found in the A. baumannii ATCC 17978 genome. Nonetheless, a role for small RNAs in iron homeostasis can not be ruled out, since the A. baumannii ATCC 17978 genome contains a gene encoding the RNA chaperone Hfq (A1S_3785), which is required for the functionality of numerous small RNAs involved in iron homeostasis in various Gram-negative bacteria $[41,42]$. The results of this study suggest that FUR is the primary regulator of iron uptake in $A$. baumannii.

Transcriptional profiling of the siderophore mediated iron acquisition mechanisms

Genes involved in the biosynthesis, efflux and uptake of a siderophore are often clustered within bacterial genomes. To date, three putative siderophore gene clusters have been identified in A. baumannii [21-23], of which two can be found in strain ATCC 17978. A significant finding from the microarray results was the detection of the novel putative A. baumannii siderophore gene cluster (Figure 2; Siderophore cluster 1: A1S_1647 A1S_1657). Several genes within siderophore cluster 1 were more than 100-fold overproduced under iron limitation, highlighting their potential importance in iron uptake (Figures 2 and 6a). Siderophore cluster 1 contains eight genes with a putative function in siderophore biosynthesis, A1S_1647, A1S_1648, A1S_1650-1654 and A1S_1657. Siderophore extrusion is most likely facilitated by an MFS efflux pump (A1S_1649). A receptor (A1S_1655) and PepSY-associated transmembrane helix family protein (A1S_1656) are likely to be involved in recognition and reduction of ferric siderophores, respectively. FUR boxes for transcriptional regulation of the unidirectional, operon-like gene cluster could be identified upstream of A1S_1647 and A1S_1657 (Figure 6a).

Siderophore gene cluster 2 (A1S_2562-2581) [23] showed similarly high levels of overexpression as cluster 1 (Figure 6b). This cluster contains 15 genes involved in siderophore biosynthesis (A1S_2567-2581), three genes involved in recognition and uptake of the ferric siderophore (A1S_2563, A1S_2564 and A1S_2566) and two genes encoding putative efflux pumps. One efflux pump gene, A1S_2565, encodes a putative MFS efflux pump. As mentioned previously, members of this family have been identified in various siderophore gene clusters in other bacteria and have proven to play a role in the efflux of enterobactin [14]. The second efflux pump, A1S_2562 is a member of the multidrug and toxic compound extrusion (MATE) family [43]. To our knowledge this is the first report of a bacterial MATE pump having a putative role in siderophore efflux. Similar to siderophore cluster 1, FUR appears to be the main transcriptional regulator, since binding sites could be identified upstream of A1S_2566, A1S_2567 and A1S_2581 (Figure 6b).

The most extensively characterized Acinetobacter siderophore gene cluster is that responsible for biosynthesis of acinetobactin [22]. Acinetobactin is synthesized from a 2,3-dihydroxybenzoic acid, threonine and hydroxyhistamine, and contains catecholate and hydroxamate groups that provide a high affinity for iron [25]. The acinetobactin biosynthesis genes include bas A-D, basF- $J$ and entE (Figure 6c). Gene pair barA and barB encodes a siderophore efflux system of the $\mathrm{ABC}$-superfamily, the products of $b a u A-F$ form a receptor for recognition of ferric acinetobactin and the products of bauB-E are involved in translocation of ferric acinetobactin (Figure 6c). All of these genes showed high levels of overexpression, ranging from 43 -fold to 165 -fold. The cluster contains putative FUR boxes upstream of basJ, entE/basD, basA/bau and bauD/basD. These same FUR binding sites have been experimentally identified using a FUR titration assay [22], validating the FUR analysis described here.

Interestingly, transcriptional up-regulation gradually decreased in all three siderophore clusters when distance from the FUR box increased, demonstrating the importance of FUR in regulating siderophore production 


\section{A. Siderophore cluster 1}

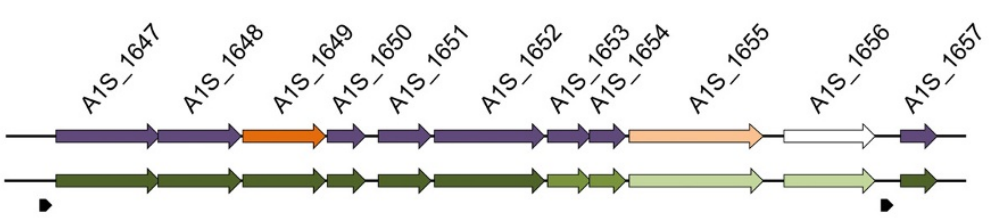

Times-fold:

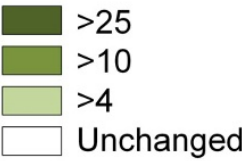

Gene function:

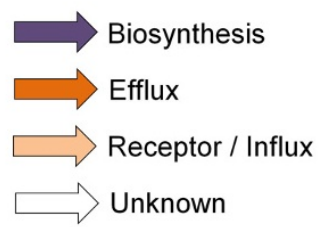

\section{B. Siderophore cluster 2}

$\overline{1 \mathrm{~Kb}} \quad$ FUR binding site

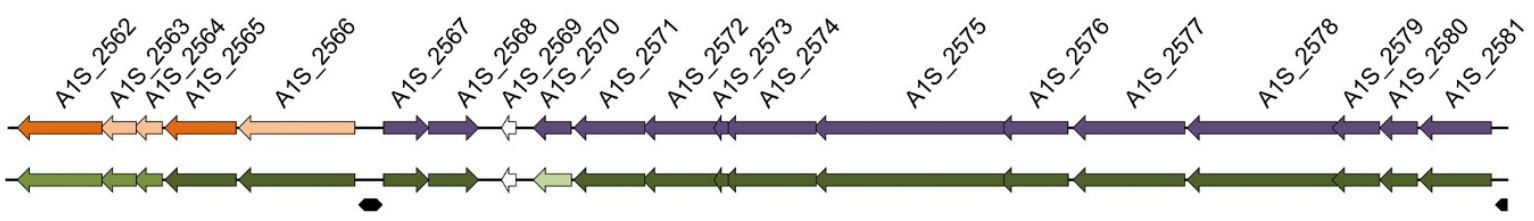

\section{Acinetobactin cluster}

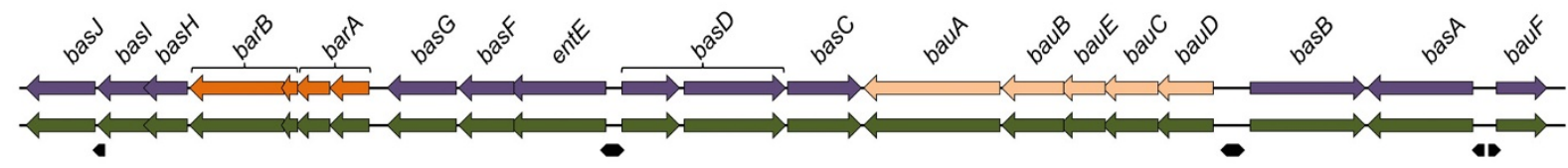

Figure 6 Transcriptional profiling of three siderophore gene clusters identified in A. baumannii ATCC 17978. Transcriptional alteration of the three siderophore gene clusters to low iron conditions are shown, (A) siderophore gene cluster 1 (A1S_1647-1657), (B) siderophore cluster 2 (A1S_2562-2581) and (C) the acinetobactin gene cluster (A1S_2372-2392). The top arrows show predicted gene function; siderophore biosynthesis in purple, receptors and uptake mechanisms in light orange, efflux pumps in orange and genes of unknown function in white. The relative transcriptional differences between A. baumannii grown under iron replete and iron limiting conditions are depicted in the bottom set of arrows according to the green color scale bar, all values are in times-fold difference. Genes depicted in white were not differentially expressed and those in dark green were overexpressed more than 25 -fold. No significant down-regulation was observed within the siderophore gene clusters. Putative FUR boxes are shown as black arrows.

at the level of gene transcription. This is the first time that a full transcriptional profile has been provided for the siderophore gene clusters in A. baumannii under iron limiting conditions. Most importantly, a novel putative siderophore gene cluster was identified.

Siderophore receptors expressed on the surface of the bacterial outer membrane play a crucial role in the recognition of iron-loaded siderophores and therefore iron uptake. These receptors are likely to recruit the TonB-ExbB-ExbD translocation system for transport of ferric siderophores from the extracellular space to the cytoplasm [16,44]. The A. baumannii ATCC 17978 genome contains 22 putative siderophore receptors. Analysis demonstrated that 15 of these are located downstream of a putative FUR box (Additional file 2) of which 11 were significantly up-regulated under iron limiting conditions.

Under iron limited conditions, high levels of overexpression were also determined for the tonB-exbB-exbD gene cluster (A1S_0452-0454) which contained a predicted FUR box. Previously, a second TonB-ExbB-ExbD energy transduction system in strain ATCC 17978 (A1S_1603-1605) was described [23]. The cluster restored enterobactin utilization in E. coli exbBD mutants, but not in tonB mutants. However, in our study, no significant transcriptional up-regulation of the genes within this cluster (A1S_1603-1613) was observed under iron limiting conditions. It is possible that the cluster is related to heme acquisition rather than siderophore mediated iron uptake, since genes related to hemophore utilization were located adjacent to this cluster. A heme receptor/reduction mechanism may not be required under the conditions tested in our study, since no hemophores are being synthesized by $A$. baumannii ATCC 17978 and no exogenous hemophores were present.

Investigation of motility under iron limiting conditions It is well established that pili play an important role in the pathogenicity of bacteria due to their roles in motility, adherence, invasion and resistance $[45,46]$. Grouping transcriptome results from this study by COG function showed that $18 \%$ of the genes related to motility were significantly down-regulated under iron limiting conditions (Figure 4). The down-regulated genes from this group are part of the chaperone-usher pili assembly systems (type I pili) and type IV pili that have been previously identified in A. baumannii [47]. 
Homologous features have been associated with biofilm formation and motility in various organisms, including E. coli and P. aeruginosa [48-50].

In $A$. baumannii, biofilm formation on abiotic surfaces

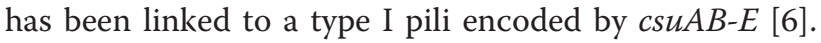
The CsuA/B, CsuA, CsuB and CsuE proteins are predicted to form part of the type I pili rod $[6,46]$. CsuC forms a periplasmic chaperone protein that accelerates folding of the pilus rod subunits and CsuD is an outer membrane protein (OMP) responsible for assembly and extension of the pilus $[6,46]$. CsuD shares $40 \%$ and $45 \%$ amino acid sequence similarity with the OMP of two other type I pili mechanisms in strain ATCC 17978, A1S_1508 and A1S_2089, respectively. csuC and homo$\log$ A1S_1509 were both down-regulated under iron starvation, by 2.0-fold and 3.7-fold, respectively (Figure 7a). The $\operatorname{csuB}$ and $\operatorname{csuE}$ homologs within this second cluster, A1S_1507 and A1S_1510, respectively, were also significantly down-regulated under iron limitation. There were no genes down-regulated in the third type I pili cluster (A1S_2088-2091). It has been shown that transcription of the csu cluster in A. baumannii is controlled by the BfmRS two-component regulator [51]. However, no significant differential expression was observed for either gene encoding this system in this study. Biofilm assays were performed under iron replete and iron limited conditions in order to assess the impact of down-regulation of the csu cluster in the formation of these structures. However, no significant differences were observed between planktonic growth and biofilm formation under iron limiting or replete conditions (data not shown). A similar study with P. aeruginosa on the effect of iron limitation on biofilm formation showed that growth as a biofilm was impaired to greater extent than planktonic growth [52]. Interestingly, it was also shown that twitching motility was enhanced when iron was less readily available [52], which could correlate with the overexpression of the type IV pili observed in Moraxella catarrhalis under iron limitation [53]. These findings indicate that binding and adherence characteristics follow different regulatory pathways in $A$. baumannii.

Various genes involved in the biosynthesis of type IV pili, including pilB-D, pilT, pilU, comM-O, comL, comQ and genes that play a role in chemosensory and regulation of this complex, pilG-J, pilR, pilS and the chpA-like, were down-regulated under iron limitation in strain ATCC 17978 (Figure 7b). In P. aeruginosa, type IV pili have proven to play a role in swarming motility, a form of migration over nutrient rich semi-solid surfaces $[54,55]$. Previous studies on the type IV secretion mechanism in Acinetobacter have been predominantly related to its function in DNA acquisition [56,57]. A swarming phenotype on Luria-Bertani medium containing a low percentage $(0.25 \%)$ of agar was determined for A. baumannii strain ATCC 17978 (Eijkelkamp et al., unpublished data). The effect of iron limitation on swarming motility was investigated by supplementation of DIP to the swarming medium. Strain ATCC 17978 was found to be incapable of migrating over the surface of the semi-solid medium when 200 $\mu \mathrm{M}$ DIP was supplemented in the medium, whereas non-migrational growth remained largely unchanged (Figure 8). Since bacterial motility is a high energy consuming process, the inability to migrate may be a stress response of $A$. baumannii ATCC 17978 to low iron levels.

\section{Comparative analysis of the iron acquisition mechanisms of sequenced Acinetobacter isolates}

The iron uptake machinery encoded by different $A$. baumannii strains may differ, as variation in the composition of siderophore mediated iron uptake proteins in the outer-membrane has been shown in a study on different Acinetobacter strains [20]. Moreover, a siderophore gene cluster found in A. baumannii 8399 (om73 - entD) could not be identified in any other sequenced Acinetobacter strain [21]. To explore this possibility in more detail comparative analyses of siderophore gene clusters were conducted using 10 fully sequenced Acinetobacter genomes, including $A$. baumannii strains ATCC 17978, ATCC 19606, AYE, AB0057, ACICU, 307-0294, D1279779, WM99c and SDF, and A. baylyi strain ADP1.

The novel putative siderophore cluster 1 (A1S_16471657) was found to be well conserved between strains ATCC 17978, ATCC 19606, AYE, AB0057, ACICU, 307-0294, D127 and WM99c (Table 2). Interestingly, the boundaries of this cluster, including genes orthologous to A1S_1647 and A1S_1657 that encode proteins with homology to the siderophore biosynthesis proteins IucA/IucC and acetyltransferase, respectively, were identified in strain SDF but the intervening genes appear to have been replaced by a $3.5 \mathrm{~kb}$ transposon encoding a transposase of the IS5 family (Figure 9). Well over 100 copies of this insertion sequence are found throughout the SDF genome and, along with other insertion sequence elements, are known to have played a major role in genome reduction in this environmental $A$. baumannii strain [47]. No other putative siderophore biosynthesis gene clusters were identified in strain SDF. Therefore, as a result of this insertion, this strain does not appear to encode any siderophore mediated iron acquisition mechanisms. This may be one reason that this strain has a higher requirement for soluble iron than other A. baumannii strains, as outlined below. Cluster 1 could also be found in A. baylyi APD1, however, it lacked a putative membrane protein (A1S_1656) 


\section{A. Type I}
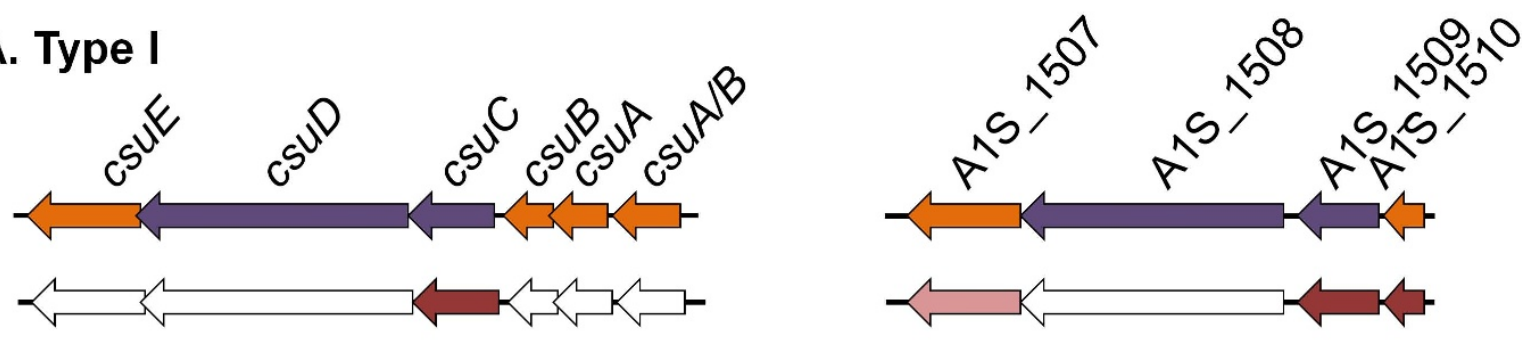

\section{B. Type IV}
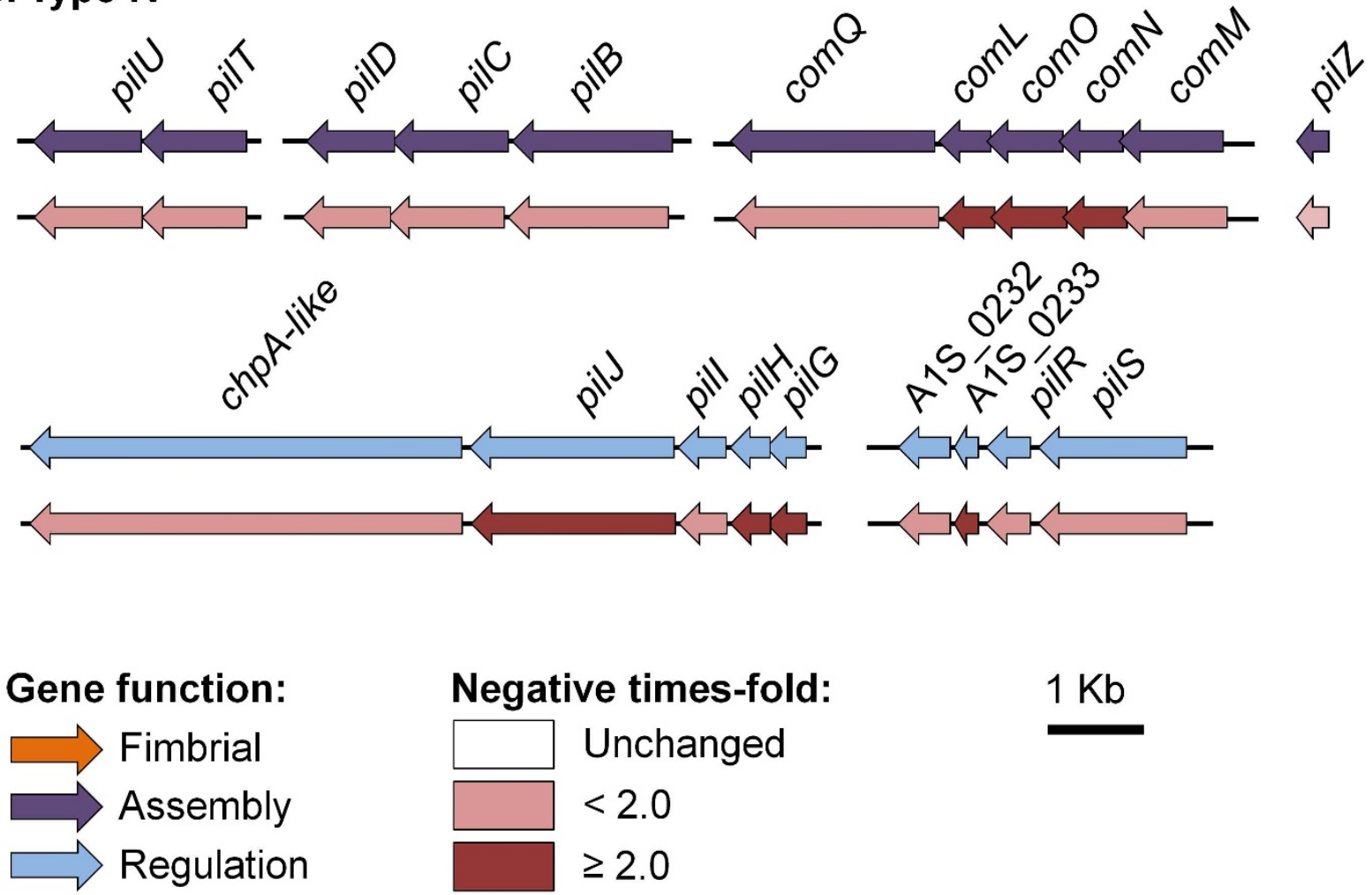

Unes-fold:

Figure 7 Gene clusters with a putative role in motility. Many genes in the motility COG were found down-regulated including genes of (A) type I and (B) type IV pili. The top set of arrows show predicted gene function; pilus proteins in orange, pilus assembly proteins in purple, and regulatory proteins in light blue. The relative transcriptional differences between A. baumannii grown under iron rich and iron limiting conditions is depicted in the bottom set of arrows, all values are in times-fold. Genes depicted in white were not differentially expressed, those shaded in light red were down-regulated less than 2-fold, whereas those in dark red were down-regulated 2-fold or more. No significant up-regulation was observed within gene clusters related to motility.

and acetyltransferase (A1S_1657). Instead, the ADP1 cluster contained a putative acetyltransferase (ACIAD2117) inserted between orthologs of A1S_1654 and A1S_1655 (Figure 9). The putative membrane protein A1S_1657 is most likely involved in recognition of the chelated siderophore, however, this role could be fulfilled by other ferric siderophore receptors, encoded distally in the genome. Whereas, cluster 1 is in the same genomic position in all $A$. baumannii strains, in $A$. baylyi ADP1 it appears to have been subjected to genomic rearrangement as it is located in a different position. Of the 10 Acinetobacter strains surveyed, siderophore cluster 2 was only detected in ATCC 17978 and ADP1. Additionally, no positive hits for A1S_2562, a gene within this siderophore cluster, were identified in 59 clinical Acinetobacter isolates from widespread locations in Australia using PCR screening (data not shown). Therefore, this cluster appears to be relatively rare 


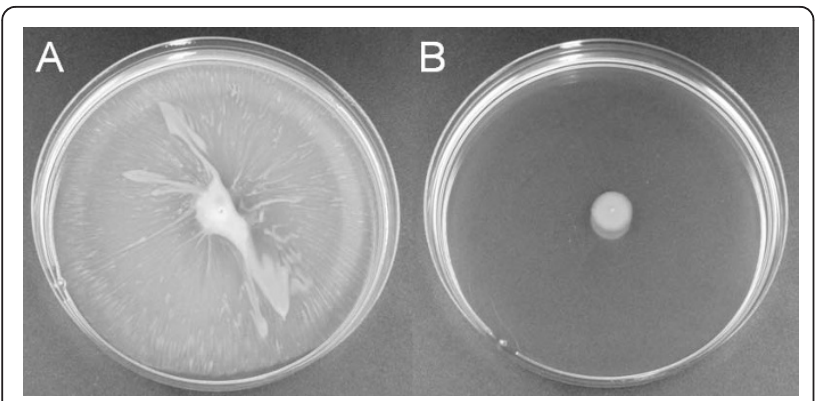

Figure 8 Swarming of $A$. baumannii ATCC 17978. A. baumannii colony material was spotted on Luria-Bertani medium containing $0.25 \%$ agar. Swarming motility (A) is visible as the channel-like growth around the dense white colony material. The absence of halo growth around the colony (B) indicates lack of swarming motility of $A$. baumannii when available iron is limited.

across the Acinetobacter genus. The average homology between the ATCC 17978 and ADP1 siderophore cluster 2 genes was 75\%, which is high compared to orthologous genes elsewhere within these two genomes, e.g. $53 \%$ within siderophore cluster 1 . Transposases were found at the termini of siderophore cluster 2 in both ATCC 17878 and ADP1, suggesting that this gene cluster may have been horizontally acquired. Nonetheless, these transposases are distinct and have inserted into distinct genomic positions in the two strains, suggesting that siderophore cluster 2 was incorporated into the two genomes in separate transfer events. No other hits were obtained in a BLASTn search of the GenBank database with cluster 2, therefore, the origin of this cluster remains unknown.

A high level of conservation was observed for the acinetobactin gene cluster among most $A$. baumannii isolates (Table 2). However, this cluster was not seen in the SDF and ADP1 strains. A fifth cluster was identified by BLASTp searches in several sequenced $A$. baumannii strains (considering the $A$. baumannii 8399 siderophore cluster as the fourth Acinetobacter siderophore gene cluster) (Table 2). The genes are represented in strain AYE by ABAYE1888 and ABAYE1889. A putative FUR box could be identified upstream of ABAYE1889 and high expression levels were observed under iron limiting conditions using qRT-PCR analysis (data not shown). The two genes encode proteins that were found to be homologous to an isochorismatase and a 2,3-dihydro2,3-hydroxybenzoate dehydrogenase. The product synthesized by these two enzymes in this cluster, 2,3dihydroxybenzoate, is an iron binding compound, but also a precursor component for more complex siderophores, like acinetobactin. This cluster is well conserved between strains AYE, AB0057, ACICU, 307-0294, ATCC 19606, D1279779 and WM99c.

The minimum inhibitory concentration (MIC) of DIP was determined for seven of the strains included in the genetic comparison (A. baumannii strains ATCC 17978, ATCC 19606, AYE, D1279779, WM99c and SDF, and $A$. baylyi strain ADP1). Growth of A. baylyi strain ADP1 and A. baumannii SDF was inhibited at lower levels compared to other strains. ATCC 17978 did not show higher MIC values for DIP compared to AYE, WM99c or D1279779, despite having three highly expressed siderophore gene clusters. Therefore, viability of Acinetobacter strains under varying iron concentrations does not appear to directly correlate with the presence or absence of siderophore gene clusters. Strains with higher $\mathrm{OD}_{600}$ values under iron replete conditions showed higher MIC levels for DIP.

\section{A second FUR-like transcription repressor}

Various bacterial genomes contain multiple FUR-like genes. These FUR homologs often encode repressors with similar domains but with higher affinity for metals

Table 2 Genomic comparison of siderophore gene clusters in sequenced Acinetobacter isolates

\begin{tabular}{|c|c|c|c|c|c|c|}
\hline & & $\begin{array}{c}\text { Cluster } 1 \\
\text { A1S_1647-1657 }\end{array}$ & $\begin{array}{c}\text { Cluster } 2 \\
\text { A1S_2562-2581 }\end{array}$ & $\begin{array}{l}\text { Acinetobactin } \\
\text { A1S_2372-2392 }\end{array}$ & $\begin{array}{l}\text { Cluster } 4 \\
\text { om73-entD }\end{array}$ & $\begin{array}{c}\text { Cluster } 5 \text { ABAYE1888 and } \\
\text { ABAYE1889 }\end{array}$ \\
\hline \multirow[t]{10}{*}{$\begin{array}{l}\text { A. } \\
\text { baumannii }\end{array}$} & $\begin{array}{l}\text { ATCC } \\
17978\end{array}$ & + & + & + & - & - \\
\hline & $\begin{array}{l}\text { ATCC } \\
19606\end{array}$ & + & - & + & - & + \\
\hline & AYE & + & - & + & - & + \\
\hline & AB0057 & + & - & + & - & + \\
\hline & $\mathrm{ACICU}$ & + & - & + & - & + \\
\hline & $307-0294$ & + & - & + & - & + \\
\hline & D1279779 & + & - & + & - & + \\
\hline & WM99c & + & - & + & - & + \\
\hline & SDF & - & - & - & - & - \\
\hline & 8399 & nd & nd & nd & + & nd \\
\hline A. baylyi & ADP1 & + & + & - & - & - \\
\hline
\end{tabular}

nd $=$ not determined. 


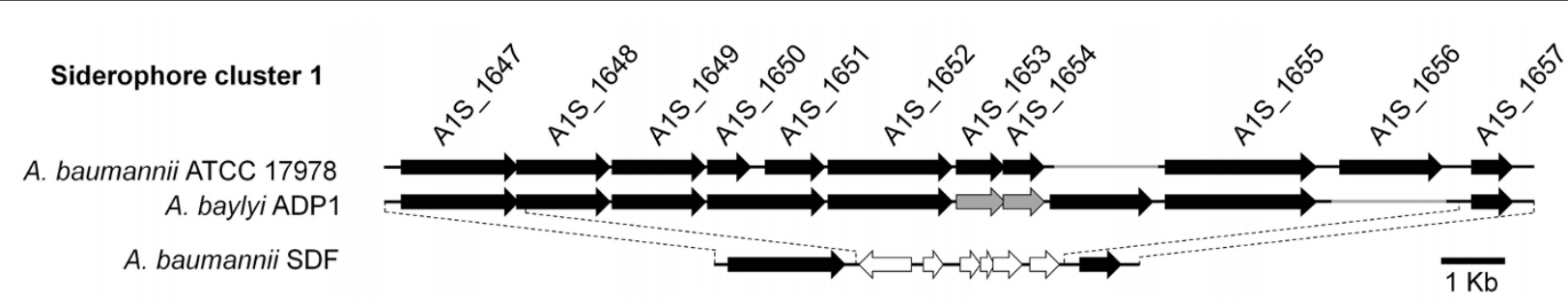

Figure 9 Comparison of siderophore cluster 1 in sequenced Acinetobacter isolates. The alignment of siderophore cluster 1 between strain ATCC 17978, ADP1 and SDF. Arrows indicate open reading frames, in black genes with high homology (> 50\% identity) and in grey genes with low homology (<50\% identity). White genes represent a $3.5 \mathrm{~Kb}$ transposon insertion, which replaced the A1S_1648-1656 orthologs in strain SDF.

other than iron, such as zinc, manganese or nickel [58]. The A. baumannii AYE genome also encoded a second FUR-like regulator that has not yet been characterized, ABAYE1887. This gene was located adjacent to the putative siderophore genes described above (ABAYE1888 and ABAYE1889). Conserved domain (CD)-searches showed the highest homology with cd07153 $\left(E=1 \mathrm{e}^{-10}\right)$, which can be found in FUR and other metalloregulatory proteins. A putative zinc uptake regulator (ZUR) can be found in a zinc-uptake gene cluster in strain AYE (ABAYE3726). Pairwise alignment of ABAYE1887 and FUR (ABAYE2920), and ABAYE1887 and ZUR, indicated higher homology for ABAYE1887 to FUR than to ZUR, showing $46 \%$ and $33 \%$ similarity, respectively. Little is known about autoregulation of FUR homologs. In the case of ABAYE1887, a FUR box with a low p-value can be found less than 200 bp upstream of the start codon using the ATCC 17978 optimized FUR motif. Moreover, qRT-PCR analysis demonstrated that growth of $A$. baumannii AYE under iron limiting conditions resulted in approximately 164-fold up-regulation of ABAYE1887, whereas, FUR only showed 1.6-fold up-regulation. This second FUR-like gene can also be found in strains ATCC 19606, D1279779 and WM99c, and a truncated form in SDF. Further experimental work is required to determine if ABAYE1887 plays a role in transcription of genes related to iron acquisition.

\section{Conclusions}

This study defined the global transcriptional response of A. baumannii to iron starvation. The up-regulation of three siderophore mediated iron acquisition systems was the predominant feature of this transcriptional response. The high level of overexpression of these systems under iron limitation, suggests that each is active in mediating iron uptake and therefore likely to be of importance to A. baumannii for survival in iron limited environments, such as human hosts. Several genes involved in other processes, such as respiration and electron transport were also significantly differentially expressed. Our data corroborate results from a recently published proteomic study of $A$. baumannii under iron rich and iron limiting conditions [59], such as up-regulation of the iron acquisition mechanisms and fumC, and the down-regulation of fumA and ompW. The abundance of putative FUR binding sites identified upstream of up-regulated genes highlighted a major role for this regulator in transcriptional up-regulation under iron limiting conditions. Various genes of the type IV pili were down-regulated under iron limiting conditions. This may in fact explain the inability of strain ATCC 17978 to migrate on semisolid surfaces under low iron concentrations. Overall, the results indicated that $A$. baumannii is adaptable to an environment with limiting iron availability.

\section{Methods}

Bacterial strains and growth conditions

Acinetobacter strains were obtained from the following sources: ATCC 17978 [GenBank: NC_009085] and 19606 [GenBank: NZ_ACQB00000000] from the American Type Culture Collection (ATCC); SDF [GenBank: NC_010400] from the Collection de Souches de l'Unité des Rickettsies (CSUR), Marseille, France; AYE [GenBank: NC_010410] from Patrice Nordmann, Dept. Bacteriologie-Virologie, Hopital de Bicetre, Le-KremlinBicetre, France; WM99c and ADP1 [GenBank: NC_005966] from Jon Iredell, Westmead Millennium Institute, Sydney, Australia; and D1279779 from The Menzies School of Health Research, Darwin, Australia. The ATCC 19606 genomic sequence was obtained from the NCBI REFSEQ database. A. baumannii strains D1279779 and WM99c were sequenced recently by 454 pyrosequencing (Paulsen et al., unpublished data). These Whole Genome Shotgun projects have been deposited at DDBJ/EMBL/GenBank under the accession numbers AERY00000000 for WM99c and AERZ00000000 for D1279779. The versions described in this paper are the first versions, AERY01000000 and AERZ01000000, respectively. The scaffold sequences of these three strains were tiled to the ATCC 17978 genome using Mauve [60]. 
Iron limitation was achieved by growing Acinetobacter strains in Mueller-Hinton (MH) medium supplemented with 2,2'-dipyridyl (DIP). The growth curves were obtained by culturing A. baumannii ATCC 17978 with different concentrations of available iron in MuellerHinton (MH) medium; untreated, $100 \mu \mathrm{M}$ DIP, $200 \mu \mathrm{M}$ DIP and $300 \mu \mathrm{M}$ DIP. In all following experiments, cultures in $\mathrm{MH}$ were supplemented with $200 \mu \mathrm{M}$ DIP.

\section{Microarray development}

An $8 \times 15 \mathrm{~K}$ custom genomic microarray was developed for A. baumannii ATCC 17978 on the Agilent platform using the Agilent eArray package http://earray.chem. agilent.com/earray/. At least four 60 mer DNA oligonucleotides with an average GC \% of 41.5, were incorporated into the design for each of the protein coding genes annotated in the ATCC 17978 genome sequence [61]. The array also included a set of intra array controls; 132 probes replicated at least 10 times in the design, and the Agilent control spots.

\section{RNA isolation}

Cells cultured under iron replete, untreated $\mathrm{MH}$, and iron limited conditions of MH with $200 \mu \mathrm{M}$ DIP, were grown until they reached mid-log phase $\left(\mathrm{OD}_{600}=0.7\right)$. The cells were pelleted and lyzed in $1 \mathrm{~mL} \mathrm{TRIzol}{ }^{\circledR}$ reagent (Invitrogen, Australia). Following phase separation, RNA was extracted from the aqueous phase using the PureLink ${ }^{\mathrm{TM}}$ Micro-to-Midi Total RNA Purification kit (Invitrogen), incorporating an oncolumn DNAseI (Invitrogen) digestion, as per manufacturer's recommendations.

\section{cDNA synthesis and microarray hybridization}

For the microarray analyses, the cDNA synthesis, labelling and hybridizations were conducted at the Ramaciotti Centre for Gene Function Analysis, University of NSW, Australia. Total RNA was reverse transcribed and labelled with either Cy3 or Cy5 using the Agilent Fairplay Microarray Labelling kit (Stratagene). Labelled cDNA samples were hybridized to a custom designed $8 \times 15 \mathrm{~K}$ two colour gene expression microarray slide. The results reported are based on three biological and four technical repeats, including one dye-swap experiment. Statistical analysis was performed on $\log _{2}$ transformed signal ratios of the replicate spots using the SAM algorithms [27]. All results described were found to be significant using a false discovery rate of less than $5 \%$ unless otherwise indicated. All microarray data presented are in accordance with the Microarray Gene Expression Data Society's minimum information about microarray experiment recommendations [62]. Descriptions of the microarray experiments, quantification data and array design have been deposited into GEO http://www.ncbi.nlm.nih.gov/geo/ and can be accessed using the accession number GSE24921.

\section{Quantitative RT-PCR}

Validation of the microarray results, preliminary experiments with FUR and transcription level measurements of A. baumannii strain AYE were performed using a two-step quantitive RT-PCR (qRT-PCR). RNA isolation was performed as described above. cDNA was synthesized using random hexamers and SuperscriptII (Invitrogen). Primers were designed to generate 100 - $150 \mathrm{bp}$ amplicons and are listed in Table 3. qPCR was performed using Sybr Green mastermix (Invitrogen). Transcriptional differences were calculated using the $\Delta \Delta C_{t}$ method [63].

\section{A. baumannii FUR binding site analysis}

A scoring matrix was defined from the 48 experimentally determined $E$. coli FUR binding sites [34] using the Multiple Em for Motif Elicitation (MEME) tool. The $A$. baumannii ATCC 17978 genome was analysed with the resulting scoring matrix using the Motif Alignment and Search Tool (MAST). Putative FUR binding site sequences that are located within the $200 \mathrm{bp}$ region upstream a start codon, with a p-value of less than $10 \mathrm{e}^{-5}$ and of which the downstream gene showed more than 4-fold up-regulation were further investigated. Subsequent MEME and MAST analyses with the described criteria were performed until no new positive hits were obtained. The resulting 21 putative FUR binding site

\section{Table 3 Oligonucleotides used in the study}

\begin{tabular}{|c|c|c|}
\hline Locus tag & Forward primer $\left(5^{\prime}-3^{\prime}\right)$ & Reverse primer $\left(5^{\prime}-3^{\prime}\right)$ \\
\hline A1S_rO1*+ & CAGCTCGTGTCGTGAGATGT & CGTAAGGGCCATGATGACTT \\
\hline A1S_2501 ${ }^{+}$ & CAACACTGGTAAATGGCGTG & ACAACGTITTCATITCGCC \\
\hline A1S_0395 & TCATGCTCTTGTTCAGTGGC & GCATTGCCAATACCCCTAGA \\
\hline A1S_3420 & GCCTTGCTITACTTGTTCCG & GCATCAGTAAATGGGCAGGT \\
\hline A1S_2562 & TTGCCATCAGTAGTGCAACC & TCCTGCAATCACAACACCAT \\
\hline A1S_3371 & CAGATCCAACTGTGGTGGTG & TCAGCATCGGTACGGTTACA \\
\hline A1S_0895 & GCGCAAAGCTGGACTTAAAG & CGGTAAACTGTCGCAAGTCC \\
\hline A1S_2565 & TGGCTCGATATTCAACGTCA & TAACAGCAAACCACCACCAA \\
\hline A1S_0897 & CCGCGAGCGACTAAGC & TGTCGCAGCCCATGAA \\
\hline A1S_1647 & GGACGCCATCGTCTCG & GCGTCCCGGCTITGTA \\
\hline A1S_1925 & GGTGGCGCGCTATTTG & GTTGCGCCATTGGGTA \\
\hline A1S_2080 & GGTCGATGGCGTTCCA & CAGCCGCTITCGTGGT \\
\hline A1S_3195 & GCGCTCAACCGCGTAA & TGCCGGATCGTCTTGC \\
\hline A1S_1509 & CCAAGGAAGGCGCTGT & TTGGGGAATGGCTTGC \\
\hline ABAYE1887 & CCCTITGATGATITACGG & CAAGGCTTAAGCGCGGTA \\
\hline ABAYE1888 & CCAGCGCATCACCACA & TCCGCTCGAACAACTCA \\
\hline ABAYE1889 & GGGGCGATTCAAGTGC & TCGCGATCAGCCAACA \\
\hline
\end{tabular}

* Primer sequences obtained from Higgins et al., 2004 [66].

${ }^{+}$Oligonucluotides used as references. 
sequences were aligned using Weblogo 3.0 [64] to create the optimized A. baumannii FUR motif.

\section{Swarming motility assay}

Swarming motility assays were performed at $37^{\circ} \mathrm{C}$ on Luria Bertani (LB) medium containing 0.25\% agar. Positive swarmers showed a halo growth zone with channellike structures.

\section{Static biofilm formation assay}

The static biofilm formation assay was performed as described previously [65] with minor modifications. $\mathrm{MH}$ broth was inoculated with bacterial colony material and incubated overnight at $37^{\circ} \mathrm{C}$. The cultures were subsequently diluted 1:100 in fresh $\mathrm{MH}$ broth in polystyrene microtiter trays and incubated $\mathrm{ON}$ at $37^{\circ} \mathrm{C}$. Adherent cells were washed once with PBS, stained by incubation with $0.1 \%$ crystal violet for $30 \mathrm{~min}$ at $4{ }^{\circ} \mathrm{C}$, and washed $3 \mathrm{X}$ with PBS. Dye was released from the cells using ethanol:acetone $(4: 1)$ and shaking at 200 $\mathrm{rpm}$ for $30 \mathrm{~min}$ at RT. Absorbance was measured at $595 \mathrm{~nm}$ on a Fluostar Omega spectrometer (BMG Labtech, Offenburg, Germany). The biofilm data represent the average of at least 3 independent experiments of triplicate wells.

\section{Additional material}

Additional file 1: Significance analysis of the microarray results. Significant differentially expressed genes in the microarray, determined by SAM [27]

Additional file 2: ATCC 17978 FUR binding sites. A list of putative FUR binding sites in the A. baumannii ATCC 17978 genome determined by MAST with $\mathrm{E}<100$

\section{Acknowledgements}

We would like to thank Patrice Nordmann (Hopital de Bicetre), Jon Iredell (Westmead Millennium Institute) and The Menzies Darwin for their kind gift of the Acinetobacter isolates. This project was financially supported by the National Health and Medical Research Council Australia, Project Grant 535053 to MHB and ITP. ITP is the recipient of a Life Science Research Award from the NSW Office of Science and Medical Research. BAE is the recipient of a School of Biological Sciences Endeavour International Postgraduate Research Scholarship.

\section{Author details}

'School of Biological Sciences, Flinders University, Adelaide, SA 5001, Australia. ${ }^{2}$ Department of Chemistry and Biomolecular Sciences, Macquarie University, Sydney, NSW 2109, Australia.

\section{Authors' contributions}

$\mathrm{BAE}, \mathrm{KAH}$, ITP and MHB designed the research project. BAE and $\mathrm{KAH}$ carried out the experiments and $B A E, K A H$, ITP and MHB wrote the manuscript. All authors have read and approved the final manuscript.

Received: 1 November 2010 Accepted: 23 February 2011

Published: 23 February 2011
References

1. Dijkshoorn $\mathrm{L}$, Nemec $\mathrm{A}$, Seifert $\mathrm{H}$ : An increasing threat in hospitals: multidrug-resistant Acinetobacter baumannii. Nat Rev Microbiol 2007, 5:939-951.

2. Falagas $M E$, Karveli EA: The changing global epidemiology of Acinetobacter baumannii infections: a development with major public health implications. Clin Microbiol Infect 2007, 13:117-119.

3. Cunha BA, Klimek JJ, Gracewski J, McLaughlin JC, Quintiliani R: A common source outbreak of Acinetobacter pulmonary infections traced to Wright respirometers. Postgrad Med J 1980, 56:169-172.

4. Neely AN: A survey of gram-negative bacteria survival on hospital fabrics and plastics. J Burn Care Rehabil 2000, 21:523-527.

5. Patterson JE, Vecchio J, Pantelick EL, Farrel P, Mazon D, Zervos MJ, Hierholzer WJ Jr: Association of contaminated gloves with transmission of Acinetobacter calcoaceticus var. anitratus in an intensive care unit. Am J Med 1991, 91:479-483.

6. Tomaras AP, Dorsey CW, Edelmann RE, Actis LA: Attachment to and biofilm formation on abiotic surfaces by Acinetobacter baumannii: involvement of a novel chaperone-usher pili assembly system. Microbiology 2003, 149:3473-3484.

7. Ramirez MS, Don M, Merkier AK, Bistue AJ, Zorreguieta A, Centron D, Tolmasky ME: Naturally competent Acinetobacter baumannii clinical isolate as a convenient model for genetic studies. J Clin Microbiol 2010, 48:1488-1490.

8. Adams MD, Goglin K, Molyneaux N, Hujer KM, Lavender H, Jamison Jل MacDonald IJ, Martin KM, Russo T, Campagnari AA, et al: Comparative genome sequence analysis of multidrug-resistant Acinetobacter baumannii. J Bacteriol 2008, 190:8053-8064.

9. Fournier PE, Vallenet D, Barbe V, Audic S, Ogata H, Poirel L, Richet H, Robert C, Mangenot S, Abergel C, et al: Comparative genomics of multidrug resistance in Acinetobacter baumannii. PLoS Genet 2006, 2:e7.

10. Koster W: Cytoplasmic membrane iron permease systems in the bacterial cell envelope. Front Biosci 2005, 10:462-477.

11. Wandersman C, Delepelaire P: Bacterial iron sources: from siderophores to hemophores. Annu Rev Microbiol 2004, 58:611-647.

12. Haq RU, Wereley JP, Chitambar CR: Induction of apoptosis by iron deprivation in human leukemic CCRF-CEM cells. Exp Hematol 1995, 23:428-432.

13. Miethke M, Marahiel MA: Siderophore-based iron acquisition and pathogen control. Microbiol Mol Biol Rev 2007, 71:413-451.

14. Furrer JL, Sanders DN, Hook-Barnard IG, McIntosh MA: Export of the siderophore enterobactin in Escherichia coli: involvement of a $43 \mathrm{kDa}$ membrane exporter. Mol Microbiol 2002, 44:1225-1234.

15. Miethke M, Schmidt S, Marahiel MA: The major facilitator superfamily-type transporter YmfE and the multidrug-efflux activator Mta mediate bacillibactin secretion in Bacillus subtilis. J Bacteriol 2008, 190:5143-5152.

16. Braun V: Energy-coupled transport and signal transduction through the gram-negative outer membrane via TonB-ExbB-ExbD-dependent receptor proteins. FEMS Microbiol Rev 1995, 16:295-307.

17. Moeck GS, Coulton JW: TonB-dependent iron acquisition: mechanisms of siderophore-mediated active transport. Mol Microbiol 1998, 28:675-681.

18. Plessner O, Klapatch T, Guerinot ML: Siderophore Utilization by Bradyrhizobium japonicum. Appl Environ Microbiol 1993, 59:1688-1690.

19. Poole K, McKay GA: Iron acquisition and its control in Pseudomonas aeruginosa: many roads lead to Rome. Front Biosci 2003, 8:d661-686.

20. Dorsey CW, Beglin MS, Actis LA: Detection and analysis of iron uptake components expressed by Acinetobacter baumannii clinical isolates. J Clin Microbiol 2003, 41:4188-4193.

21. Dorsey CW, Tolmasky ME, Crosa JH, Actis LA: Genetic organization of an Acinetobacter baumannii chromosomal region harbouring genes related to siderophore biosynthesis and transport. Microbiology 2003, 149:1227-1238

22. Mihara K, Tanabe T, Yamakawa Y, Funahashi T, Nakao H, Narimatsu S, Yamamoto S: Identification and transcriptional organization of a gene cluster involved in biosynthesis and transport of acinetobactin, a siderophore produced by Acinetobacter baumannii ATCC 19606T. Microbiology 2004, 150:2587-2597

23. Zimbler DL, Penwell WF, Gaddy JA, Menke SM, Tomaras AP, Connerly PL, Actis LA: Iron acquisition functions expressed by the human pathogen Acinetobacter baumannii. Biometals 2009, 22:23-32. 
24. Dorsey CW, Tomaras AP, Connerly PL, Tolmasky ME, Crosa JH, Actis LA: The siderophore-mediated iron acquisition systems of Acinetobacter baumannii ATCC 19606 and Vibrio anguillarum 775 are structurally and functionally related. Microbiology 2004, 150:3657-3667.

25. Yamamoto S, Okujo N, Sakakibara Y: Isolation and structure elucidation of acinetobactin, a novel siderophore from Acinetobacter baumannii. Arch Microbiol 1994, 162:249-254.

26. Daniel C, Haentjens S, Bissinger MC, Courcol RJ: Characterization of the Acinetobacter baumannii Fur regulator: cloning and sequencing of the fur homolog gene. FEMS Microbiol Lett 1999, 170:199-209.

27. Tusher VG, Tibshirani R, Chu G: Significance analysis of microarrays applied to the ionizing radiation response. Proc Natl Acad Sci USA 2001, 98:5116-5121.

28. Dallas PB, Gottardo NG, Firth MJ, Beesley AH, Hoffmann K, Terry PA, Freitas JR, Boag JM, Cummings AJ, Kees UR: Gene expression levels assessed by oligonucleotide microarray analysis and quantitative realtime RT-PCR - how well do they correlate? BMC Genomics 2005, 6:59.

29. French $C E$, Bell JM, Ward FB: Diversity and distribution of hemerythrin-like proteins in prokaryotes. FEMS Microbiol Lett 2008, 279:131-145.

30. Chenier D, Beriault R, Mailloux R, Baquie M, Abramia G, Lemire J, Appanna $\mathrm{V}$ : Involvement of fumarase $\mathrm{C}$ and NADH oxidase in metabolic adaptation of Pseudomonas fluorescens cells evoked by aluminum and gallium toxicity. Appl Environ Microbiol 2008, 74:3977-3984.

31. Bailey $\mathrm{TL}$, Elkan C: Fitting a mixture model by expectation maximization to discover mitifs in biopolymers. Proceedings of the Second International Conference on Intelligent Systems for Molecular Biology AAAI Press; 1994, 28-36.

32. Bailey $\mathrm{TL}$, Gribskov M: Combining evidence using p-values: application to sequence homology searches. Bioinformatics 1998, 14:48-54.

33. Hassan KA, Johnson A, Shaffer BT, Ren Q, Kidarsa TA, Elbourne LD, Hartney S, Duboy R, Goebel NC, Zabriskie TM, et al: Inactivation of the GacA response regulator in Pseudomonas fluorescens Pf-5 has farreaching transcriptomic consequences. Environ Microbiol 2010.

34. Stojiljkovic I, Baumler AJ, Hantke K: Fur regulon in gram-negative bacteria. Identification and characterization of new iron-regulated Escherichia coli genes by a fur titration assay. J Mol Biol 1994, 236:531-545.

35. Madsen ML, Nettleton D, Thacker EL, Minion FC: Transcriptional profiling of Mycoplasma hyopneumoniae during iron depletion using microarrays. Microbiology 2006, 152:937-944

36. Ochsner UA, Wilderman PJ, Vasil Al, Vasil ML: GeneChip expression analysis of the iron starvation response in Pseudomonas aeruginosa: identification of novel pyoverdine biosynthesis genes. Mol Microbiol 2002, 45:1277-1287.

37. Palyada $K$, Threadgill D, Stintzi A: Iron acquisition and regulation in Campylobacter jejuni. J Bacteriol 2004, 186:4714-4729.

38. Paustian ML, May BJ, Kapur V: Pasteurella multocida gene expression in response to iron limitation. Infect Immun 2001, 69:4109-4115.

39. Helmann JD: The extracytoplasmic function (ECF) sigma factors. Adv Microb Physiol 2002, 46:47-110.

40. Potvin E, Sanschagrin F, Levesque RC: Sigma factors in Pseudomonas aeruginosa. FEMS Microbiol Rev 2008, 32:38-55.

41. Gottesman S: The small RNA regulators of Escherichia coli: roles and mechanisms*. Annu Rev Microbiol 2004, 58:303-328.

42. Masse E, Majdalani N, Gottesman S: Regulatory roles for small RNAs in bacteria. Curr Opin Microbiol 2003, 6:120-124.

43. Brown MH, Paulsen IT, Skurray RA: The multidrug efflux protein NorM is a prototype of a new family of transporters. Mol Microbiol 1999, 31:394-395.

44. Braun V: Iron uptake by Escherichia coli. Front Biosci 2003, 8:s1409-1421.

45. Guerry P: Campylobacter flagella: not just for motility. Trends Microbiol 2007, 15:456-461.

46. Proft T, Baker EN: Pili in Gram-negative and Gram-positive bacteria structure, assembly and their role in disease. Cell Mol Life Sci 2009, 66:613-635.

47. Vallenet $D$, Nordmann P, Barbe V, Poirel L, Mangenot S, Bataille E, Dossat C, Gas S, Kreimeyer A, Lenoble $P$, et al: Comparative analysis of Acinetobacters: three genomes for three lifestyles. PLoS One 2008, 3: e1805.

48. Haussler S: Biofilm formation by the small colony variant phenotype of Pseudomonas aeruginosa. Environ Microbiol 2004, 6:546-551.

49. Nudleman E, Kaiser D: Pulling together with type IV pili. J Mol Microbiol Biotechnol 2004, 7:52-62.
50. Waksman G, Hultgren SJ: Structural biology of the chaperone-usher pathway of pilus biogenesis. Nat Rev Microbiol 2009, 7:765-774.

51. Tomaras AP, Flagler MJ, Dorsey CW, Gaddy JA, Actis LA: Characterization of a two-component regulatory system from Acinetobacter baumannii that controls biofilm formation and cellular morphology. Microbiology 2008, 154:3398-3409.

52. Patriquin GM, Banin E, Gilmour C, Tuchman R, Greenberg EP, Poole K: Influence of quorum sensing and iron on twitching motility and biofilm formation in Pseudomonas aeruginosa. J Bacteriol 2008, 190:662-671.

53. Luke NR, Howlett AJ, Shao J, Campagnari AA: Expression of type IV pili by Moraxella catarrhalis is essential for natural competence and is affected by iron limitation. Infect Immun 2004, 72:6262-6270.

54. Kaiser D: Bacterial swarming: a re-examination of cell-movement patterns. Curr Biol 2007, 17:R561-570.

55. Overhage J, Lewenza S, Marr AK, Hancock RE: Identification of genes involved in swarming motility using a Pseudomonas aeruginosa PAO mini-Tn5-lux mutant library. J Bacteriol 2007, 189:2164-2169.

56. Herzberg C, Friedrich A, Averhoff B: comB, a novel competence gene required for natural transformation of Acinetobacter sp. BD413: identification, characterization, and analysis of growth-phase-dependent regulation. Arch Microbiol 2000, 173:220-228

57. Porstendorfer D, Drotschmann U, Averhoff B: A novel competence gene, comP, is essential for natural transformation of Acinetobacter sp. strain BD413. Appl Environ Microbiol 1997, 63:4150-4157.

58. Hantke K: Bacterial zinc transporters and regulators. Biometals 2001, 14:239-249.

59. Nwugo C, Gaddy JA, Zimbler DL, Actis LA: Deciphering the iron response in Acinetobacter baumannii: A proteomics approach. J Proteomics 2010.

60. Darling AC, Mau B, Blattner FR, Perna NT: Mauve: multiple alignment of conserved genomic sequence with rearrangements. Genome Res 2004, 14:1394-1403.

61. Smith MG, Gianoulis TA, Pukatzki S, Mekalanos JJ, Ornston LN, Gerstein M, Snyder M: New insights into Acinetobacter baumannii pathogenesis revealed by high-density pyrosequencing and transposon mutagenesis. Genes Dev 2007, 21:601-614.

62. Brazma A, Vilo J: Gene expression data analysis. Microbes Infect 2001, 3:823-829

63. Livak KJ, Schmittgen TD: Analysis of relative gene expression data using real-time quantitative PCR and the 2(-Delta Delta $C(T))$ Method. Methods 2001, 25:402-408.

64. Crooks GE, Hon G, Chandonia JM, Brenner SE: WebLogo: a sequence logo generator. Genome Res 2004, 14:1188-1190.

65. O'Toole GA, Pratt LA, Watnick PI, Newman DK, Weaver VB, Kolter R: Genetic approaches to study of biofilms. Methods Enzymol 1999, 310:91-109.

66. Higgins PG, Wisplinghoff $H$, Stefanik D, Seifert H: Selection of topoisomerase mutations and overexpression of adeB mRNA transcripts during an outbreak of Acinetobacter baumannii. J Antimicrob Chemother 2004, 54:821-823.

doi:10.1186/1471-2164-12-126

Cite this article as: Eijkelkamp et al:: Investigation of the human pathogen Acinetobacter baumannii under iron limiting conditions. BMC Genomics 2011 12:126.

\section{Submit your next manuscript to BioMed Central and take full advantage of:}

- Convenient online submission

- Thorough peer review

- No space constraints or color figure charges

- Immediate publication on acceptance

- Inclusion in PubMed, CAS, Scopus and Google Scholar

- Research which is freely available for redistribution 